\title{
Análisis cualitativo de las neoformaciones en la edad preescolar
}

\author{
Qualitative analysis of new psychological formations \\ in preschool age
}

Luz del Carmen Tejeda Arroyo ${ }^{1}$

\section{RESUMEN}

La investigación muestra los resultados cualitativos de la aplicación de juego temático de roles sobre el desarrollo de las neoformaciones de la edad preescolar en una población infantil en México, con la participación de maestras y madres de familia. El enfoque histórico cultural (VIGOTSKY, 1996) sustenta la actividad de juego, considerada como rectora en esta edad psicológica. En la investigación participaron 59 niños de tercero de preescolar, los cuales pertenecen a una institución de sostenimiento público, 2 maestras de educación preescolar, 2 maestras auxiliares y 2 madres de familia. El análisis de las condiciones de desarrollo psicológico de los niños antes y después de la aplicación de juego de roles demuestra la importancia de esta actividad rectora en el surgimiento de las neoformaciones de personalidad reflexiva, actividad voluntaria e imaginación.

Palabras clave: Enfoque histórico cultural. Neoformaciones. Personalidad reflexiva. Actividad voluntaria. Imaginación.

\begin{abstract}
The research shows the qualitative results of the application of thematic role-playing on the development of new psychological formations of preschool age in a child population in Mexico, with the participation of teachers and mothers. The cultural historical approach (VIGOTSKY, 1996) supports the play activity, considered as guiding in this psychological age. The research involved 59 third-year preschool children, who belong to a public support institution, 2 pre-school teachers, 2 assistant teachers and 2 mothers. The analysis of the conditions of psychological development of the children before and after the application of role-playing shows the importance of this guiding activity in the emergence of new psychological formations of reflexive personality, voluntary activity and imagination.
\end{abstract}

Keywords: Cultural historical approach. New psychological formations. Reflexive personality. Voluntary activity. Imagination.

\footnotetext{
1 Maestra en educación especial por la Universidad Autónoma de Tlaxcala, México. ORCID: https://orcid.org/0000-0001-9110-7409. E-mail: luzkarentejeda@gmail.com.
} 


\section{RESUMO}

A pesquisa mostra os resultados qualitativos da aplicação do jogo temático de papéis sociais no desenvolvimento de novas formações psicológicas em idade pré-escolar em uma população infantil do México, com a participação de professoras e mães. A abordagem histórico-cultural (VIGOTSKY, 1996) dá suporte à atividade de jogo de papéis, considerada como norteadora nesta idade psicológica. A pesquisa envolveu 59 préescolares do terceiro ano, pertencentes a uma instituição pública, 2 professoras de educação infantil, 2 professoras assistentes e 2 mães.
A análise das condições de desenvolvimento psicológico das crianças antes e depois da aplicação do jogo de papéis mostra a importância dessa atividade norteadora no surgimento de novas formações psicológicas de personalidade reflexiva, atividade voluntária e imaginação.

Palavras chave: Abordagem histórico-cultural. Novas formações psicológicas. Personalidade reflexiva. Atividade voluntária. Imaginação.

\section{Introducción}

Las maestras de jardín de niños en México se han enfrentado a una realidad cambiante en torno a los planteamientos curriculares de la educación preescolar. En 1979 se creó el primer Programa de estudios para este nivel educativo, normado a nivel nacional (SECRETARÍA DE EDUCACIÓN PÚBLICA, 1979) y desde ese año hasta la última reforma del programa de educación preescolar 2017 (SECRETARÍA DE EDUCACIÓN PÚBLICA, 2017) se han implementado diferentes planes de estudios, donde se puede observar la sobrevaloración de las políticas públicas (GÓMEZ, 2017) antepuestas a la importancia de modelos educativos sustentados teórica y metodológicamente.

Estos cambios curriculares de planes y programas de estudios en la educación preescolar, se han dado sin explicar de manera clara a las maestras la teoría de enseñanza- aprendizaje de la cual emanan, la capacitación y la sensibilización para apropiarse de cada nuevo plan de estudios y de sus aspectos teóricos ha carecido de sistematización (SOLOVIEVA; QUINTANAR, 2010), dando como resultado un aparente cambio educativo, que se interpreta sólo en términos de política educativa.

Este ambiguo conocimiento teórico de las maestras de educación preescolar, ha provocado la aplicación de actividades para niños de este nivel educativo que, de acuerdo con los planes y programas de estudios, han sido 
consideradas como propias de la educación preescolar, sin tener certeza del sustento científico de las mismas, así se pueden mencionar actividades para crear centros de interés (SECRETARIA DE EDUCACIÓN PÚBLICA, 1979), para abordar unidades temáticas (SECRETARIA DE EDUCACIÓN PÚBLICA, 1981), para desarrollar proyectos educativos (SECRETARIA DE EDUCACIÓN PÚBLICA, 1992), actividades convertidas en estrategias básicas de aprendizaje (SECRETARIA DE EDUCACIÓN PÚBLICA, 2004), actividades para desarrollar competencias, entre otras.

Las maestras de educación preescolar han aplicado estas actividades en seguimiento de los programas de estudio, estableciendo una relación poco específica entre ellas y el juego, concebido como "una actividad creativa natural, sin aprendizaje anticipado, que proviene de la vida misma" (MENESES; MONGE, 2001, p. 113) en donde la libertad y la espontaneidad son elementos indispensables, pero desconociendo si éstas actividades libres realmente tienen la estructura necesaria para contribuir al desarrollo de los niños.

Los planteamientos teóricos del enfoque histórico cultural de Vigotsky (1995) y la teoría de la actividad han proporcionado elementos importantes para generar cambios en el sistema educativo, sus postulados, sustentados teórica y metodológicamente han representado una posibilidad para llevar a cabo el proceso de enseñanza-aprendizaje de manera sólida, mostrando la importancia de la "actividad" en cada etapa de desarrollo.

El enfoque histórico cultural de Vigotsky (1996) determina que el desarrollo no se origina a partir de aspectos exclusivamente naturales, sino que el contexto y la apropiación de la experiencia cultural determinan el estado real de desarrollo. Analiza además el proceso de enseñanza-aprendizaje como una diada dinámica, de constante reciprocidad entre niños y maestros en incidencia mutua (SOLOVIEVA; GONZÁLEZ; ROSAS; MATA; MORALES, 2020). Vigotsky explicó en sus premisas que la enseñanza determina el desarrollo y conduce hacia el mismo, pero es importante resaltar que, la premisa de Vigostky se logra solo si la enseñanza es la adecuada y organizada, Talizina, una colaboradora de 
este mismo enfoque menciona: "no toda la enseñanza conduce al desarrollo, debido a que como sea la enseñanza, será el desarrollo del niño" (TALIZINA, 2009 , p. 8), es por ello que se resalta la importancia de este enfoque en el contexto educativo, porque plantea la necesidad de organizar la enseñanzaaprendizaje desde un conocimiento profundo de la teoría y cuyas actividades derivadas deben tener coherencia con sus postulados.

Concretamente en la educación preescolar, este enfoque ha propuesto orientaciones específicas para comprender la importancia del juego y lo que esta actividad organizada desarrolla en los niños. Elkonin (1989) precisa una clara diferencia entre edad cronológica y edad psicológica, explicando que esta última se caracteriza por la actividad que el niño realiza en cada etapa de su vida, dentro de una situación social determinada. Desde el análisis del enfoque, la actividad rectora es aquella que garantiza el desarrollo psicológico en una etapa concreta, así, en la edad preescolar, la actividad rectora es el juego temático de roles sociales (ELKONIN, 1989), esta actividad rectora propicia la aparición de nuevas formaciones psicológicas o neoformaciones, las cuales fueron definidas por Vigotsky (1984) y son las que garantizan el desarrollo en etapas posteriores.

La actividad de juego temático de roles ha sido aplicada y comprobada en su efectividad en diferentes estudios científicos (LÁZARO; SOLOVIEVA; CISNEROS; QUINTANAR, 2009; BONILLA; SOLOVIEVA; JIMÉNEZ, 2012; GARCÍA; SOLOVIEVA; QUINTANAR, 2013; GONZÁLEZ; SOLOVIEVA, 2014; SOLOVIEVA; TEJEDA; LÁZARO; QUINTANAR, 2015; GONZÁLEZ; SOLOVIEVA, 2016), en cada uno de los cuales se puede analizar la incidencia positiva sobre el desarrollo psicológico de los niños en etapa preescolar.

El juego temático de roles, como actividad rectora, favorece la aparición de las nuevas formaciones psicológicas propias de esta edad, la aparición de las neoformaciones en la edad preescolar será la muestra de que el niño está preparado para iniciar la etapa escolar (nivel de primaria), sin embargo, si estas neoformaciones no están presentes en el niño, significará que el desarrollo 
psicológico no está consolidado y, por lo tanto, no se podrá acceder a la actividad rectora de la siguiente etapa (educación primaria) con éxito (SOLOVIEVA; QUINTANAR, 2012), la cual corresponde a la actividad de estudios.

Las neoformaciones son explicadas por Vigotsky como:

El nuevo tipo de estructura de la personalidad y de su actividad, los cambios psíquicos y sociales que se producen por primera vez en cada edad y determinan, en el aspecto más importante y fundamental, la consciencia del niño, su relación con el medio, su vida interna y externa, todo el curso de su desarrollo en el período dado (VIGOTSKY, 1996, p. 254, 255).

Estas neoformaciones de la edad preescolar han sido definidas por los seguidores de Vigotsky, entre ellas se pueden mencionar: personalidad reflexiva (ELKONIN, 1995; LEONTIEV, 2010; PETROVSKI 1986), actividad voluntaria (SALMINA; FILIMONOVA, 2001) e imaginación (DAVIDOV, 1996, 2000; DYACHENKO, 2010).

La personalidad reflexiva es de suma importancia en la etapa preescolar porque gracias a ella se logra la correlación de los motivos, los más relevantes someten a otros, se da la relación correspondiente entre el sentido más importante y menos relevante para el niño. Surge la subordinación consciente e independiente de una acción a otra, las acciones aisladas se tornan más complejas dependiendo del motivo que las subyuga, es así como adquieren un sentido consciente. De esta forma, durante el desarrollo de la personalidad, el niño va incursionando en el mundo de las relaciones humanas que lo rodean y con ello en las funciones sociales, las normas y reglas de comportamiento, surge la compasión por el otro y el sentido personal de las acciones. El aspecto concluyente de esta neoformación es la relación consiente entre el impulso del niño y la acción que él debe someter a este impulso, cuando no se observa una subordinación de motivos/acciones y cuando los impulsos están presentes en las interacciones sociales, se refleja una conducta puramente reactiva (ELKONIN, 1995).

La actividad voluntaria se refiere de manera concreta al hecho de que los niños logren en un primer momento, subordinar sus acciones a la 
instrucción del adulto y después aprendan a dirigir su propia conducta hacia objetivos establecidos y a buscar los mejores medios para alcanzarlos. El niño progresivamente aprende a dominar sus acciones al control de su voluntad. Esta neoformación es determinante porque permite al niño tener la posibilidad de dirigir su propia conducta, condición esencial que demuestra la preparación psicológica del niño para ingresar al nivel de primaria e iniciarse en el proceso de enseñanza-aprendizaje de lectura, escritura y cálculo (SALMINA; FILIMONOVA, 2001).

La característica principal de la imaginación es la separación de la realidad, cuando sobre un rasgo aislado de la realidad se construye una imagen nueva y con ello, se reconstruyen y reorganizan las representaciones mentales que ya existen, esto evidencia el funcionamiento del plano interno de acciones (DYACHENKO, 2010). La imaginación entre los niños de cuatro a cinco años favorece la planificación específica, dicho de otra forma, la planificación paso a paso de un plan de acción y su realización; los niños empiezan a construir una cadena de transformaciones proyectadas en acciones y en lenguaje externo. La posibilidad de la planeación por pasos conduce a la posibilidad de planear la creatividad verbal orientada para otras acciones como la de inventar un cuento. El desarrollo de la imaginación conducirá a la solución de problemas creativos reales, presentes en la escuela y en la vida social.

La importancia de las neoformaciones en la edad preescolar radica en que estas permitirán al niño adquirir psicológicamente lo necesario para desarrollarse efectivamente en la etapa del nivel de primaria, nivel en el que aparecen las actividades escolares de lectura, escritura y cálculo, sin las neoformaciones de la etapa preescolar, el niño se enfrentará indudablemente al fracaso y/o a la aparición de dificultades en su proceso de aprendizaje.

El presente artículo tiene la intención de presentar los resultados cualitativos en la aplicación de juego temático de roles y los beneficios que las neoformaciones aportan a los niños en edad preescolar. El estudio pretende 
seguir demostrando la efectividad del enfoque histórico cultural y difundir las contribuciones de éste en el plano educativo.

\section{Método}

\subsection{Diseño}

Para el desarrollo de la presente investigación, se eligió un enfoque cualitativo debido a que, como menciona Izcara (2014), el método cualitativo busca la comprensión de fenómenos sociales desde el punto de vista de los participantes, con el objetivo de conocer la visión que éstos tienen de la realidad social.

El análisis cualitativo trata de entender en su globalidad un hecho social concreto, por ello, bajo este enfoque, se realizó una investigación inductiva, con un diseño de investigación acción. Se eligió el diseño de investigación acción debido a que, como enuncia Kemmis (1984, en LATORRE (2005):

La investigación acción... es una forma de indagación autorreflexiva realizada por quienes participan (profesorado, alumnado, o dirección, por ejemplo) en las situaciones sociales (incluyendo las educativas) para mejorar la racionalidad y la justicia de: a) sus propias prácticas sociales o educativas; b) su comprensión sobre las mismas; y c) las situaciones e instituciones en que éstas prácticas se realizan (aulas o escuelas, por ejemplo) (KEMMIS, 1984, p. 24).

\subsection{Participantes}

En la investigación participaron dos maestras de educación preescolar, dos maestras apoyos auxiliares, dos practicantes, una madre de familia licenciada en psicología, una madre de familia licenciada en educación preescolar y 59 niños de educación preescolar divididos en dos grupos. En la tabla uno se muestra la distribución de los grupos. 
Tabla 1. Características de los participantes de acuerdo a grupos, adultos orientadores, edad y género.

\begin{tabular}{|c|c|c|c|c|c|}
\hline \multirow[t]{2}{*}{ Grupo } & \multirow[t]{2}{*}{ Adultos orientadores } & \multirow{2}{*}{$\begin{array}{l}\text { Edad de los } \\
\quad \text { niños } \\
\text { (Promedio) }\end{array}$} & \multicolumn{2}{|c|}{ Género } & \multirow[t]{2}{*}{ Tota } \\
\hline & & & Hombres & Mujeres & \\
\hline $3^{\circ} \mathrm{A}$ & $\begin{array}{l}\text { Maestra titular } \\
\text { Maestra auxiliar } \\
\text { Practicante } \\
\text { Madre de familia con } \\
\text { formación en psicología }\end{array}$ & 5.6 & 16 & 13 & 29 \\
\hline $3^{\circ} \mathbf{B}$ & $\begin{array}{l}\text { Maestra titular } \\
\text { Maestra auxiliar } \\
\text { Practicante } \\
\text { Madre de familia con } \\
\text { formación en educación } \\
\text { preescolar. }\end{array}$ & 5.4 & 19 & 11 & 30 \\
\hline
\end{tabular}

Fuente: elaboración propia.

Se incluyó a todos los participantes de cada grupo escolar, ninguno de los niños participantes presentaba alguna discapacidad o alteración neuropsicológica.

Los participantes pertenecen a una institución de educación preescolar de sostenimiento público y de turno vespertino. La escuela está ubicada en el municipio de San Pedro Cholula, en el Estado de Puebla México; cuenta con todos los servicios públicos debido a que su ubicación geográfica al centro del municipio la mantiene cercana a los servicios de seguridad, drenaje, transporte, alumbrado, agua potable y centrales de abasto. El nivel socioeconómico de las familias de los participantes es medio bajo, la actividad económica de los padres es el comercio principalmente.

\subsection{Procedimiento}

Se realizó una reunión con las autoridades directivas de la escuela y con las maestras titulares de los grupos, se presentaron los objetivos de la investigación y se obtuvo la aprobación.

La primera acción fue realizar una reunión con los padres de familia de los niños para solicitar la autorización de la participación de los niños en la 
investigación y la firma del consentimiento informado. La segunda acción fue revisar los expedientes de los alumnos para identificar a algunas madres de familia cuyo perfil profesional pudiera apoyar en la aplicación de la investigación, de esta manera se solicitó el apoyo a dos madres de familia, ambas con nivel de estudios de licenciatura, es importante resaltar que estas madres de familia no tenían hijos en tercero de preescolar.

La investigación se realizó en tres fases (ver cuadro 1):

Cuadro 1. Fases de la investigación sobre el análisis cualitativo de las neoformaciones

Fase 1

Fase 2

\section{"Plan de acción"}

- Entrevista a profundidad con las maestras titulares de los grupos para conocer los aspectos generales de la actividad de juego $\mathrm{y}$ algunos aspectos sobre las neoformaciones de sus alumnos: personalidad, actividad voluntaria e imaginación.
- Capacitación de las maestras, apoyos auxiliares y madres de familia en las principales bases teóricas del enfoque Histórico Cultural y la teoría de la actividad, así como el análisis de "juego de roles" como actividad rectora y las neoformaciones básicas de la edad preescolar.

- Aplicación de juego temático de roles una vez por semana durante nueve meses.

- Reuniones mensuales de revisión en la aplicación de juego de roles y retroalimentación de los mismos.
Fase 3

Fuente: elaboración propia.

Las entrevistas a profundidad realizadas a las maestras titulares de los dos grupos de tercero de preescolar se realizaron bajo un cuestionario que contenía preguntas sobre las categorías de análisis: juego de roles y neoformaciones en preescolar. Las preguntas estuvieron dirigidas a escuchar 
el discurso de las maestras sobre su experiencia frente a actividades de juego y de juego de roles, a partir de su experiencia.

La aplicación del juego temático de roles se realizó una vez por semana en cada grupo durante nueve meses, con un total de treinta y seis sesiones por grupo, la duración de cada uno de los juegos fue de una hora o dos, tiempo en el cual, la maestra titular, el apoyo auxiliar, la practicante y la madre de familia aplicaban actividades de juego siguiendo el orden de las etapas del mismo, como se muestra en el cuadro 2.

Cuadro 2. Etapas del juego de roles aplicadas en los grupos.

\begin{tabular}{|c|c|c|c|}
\hline Grupos & Etapa del juego de roles & Descripción de la etapa & Sesiones \\
\hline \multirow[t]{4}{*}{$\begin{array}{l}3^{\circ} \mathrm{A} y \\
3^{\circ} \mathrm{B}\end{array}$} & $\begin{array}{l}\text { Juego de representación } \\
\text { materializada (material) de acciones } \\
\text { y roles. }\end{array}$ & $\begin{array}{l}\text { El niño representa situaciones sociales } \\
\text { con ayuda de objetos que corresponden } \\
\text { a los objetos y las acciones. }\end{array}$ & $1-5$ \\
\hline & $\begin{array}{l}\text { Juego de representación } \\
\text { materializada de acciones. }\end{array}$ & $\begin{array}{l}\text { El niño retoma el rol, pero requiere } \\
\text { aún el uso de objetos concretos. }\end{array}$ & $6-11$ \\
\hline & $\begin{array}{l}\text { Juego de roles con el apoyo de } \\
\text { objetos. }\end{array}$ & $\begin{array}{l}\text { El niño es capaz de retomar uno u } \\
\text { otro rol dentro del juego pero con } \\
\text { apoyo de objetos concretos. }\end{array}$ & $12-22$ \\
\hline & Juego de roles sociales. & $\begin{array}{l}\text { Etapa más avanzada, los niños ya } \\
\text { no requieren de objetos concretos (o } \\
\text { un mínimo de ellos) para la } \\
\text { realización de los roles sociales. }\end{array}$ & $22-36$ \\
\hline
\end{tabular}

Fuente: Solovieva y Quintanar (2012)

\subsection{Instrumentos}

Los indicadores de la entrevista a profundidad fueron los mismos en la fase uno y la fase tres de la investigación, en cuadro 3 se muestran las categorías de análisis consideradas y sus correspondientes indicadores. 
Cuadro 3. Indicadores considerados en la entrevista a profundidad para maestras titulares y madres de familia de apoyo.

\begin{tabular}{|c|c|c|}
\hline \multicolumn{2}{|c|}{ Categorías de análisis } & Indicadores \\
\hline Juego de roles en & preescolar & $\begin{array}{l}\text { - Conocimiento teórico de las actividades de juego en } \\
\text { preescolar. } \\
\text { - Recurrencia de la aplicación de las actividades de juego. } \\
\text { - Conocimiento sobre juego de roles. }\end{array}$ \\
\hline \multirow[t]{3}{*}{$\begin{array}{l}\text { Neoformaciones } \\
\text { en los niños de } \\
\text { preescolar }\end{array}$} & $\begin{array}{l}\text { Personalidad } \\
\text { reflexiva }\end{array}$ & $\begin{array}{l}\text { - Establecimiento de la base de los motivos en los niños. } \\
\text { - Comprensión de normas de comportamiento en el } \\
\text { salón de clases por parte de los niños. } \\
\text { - Presencia del sentido de compasión por el otro, } \\
\text { deseo de compartir y ayudar. } \\
\text { - Aspectos que evidencian el sentido personal de los niños. }\end{array}$ \\
\hline & $\begin{array}{l}\text { Actividad } \\
\text { voluntaria }\end{array}$ & $\begin{array}{l}\text { - Subordinación de las acciones de los niños a la } \\
\text { instrucción del adulto. } \\
\text { - Indicios sobre la autodirección de la propia conducta } \\
\text { de los niños hacia objetivos establecidos y los medios } \\
\text { para alcanzarlos. } \\
\text { - Uso del lenguaje en su función reguladora. } \\
\text { - Control y organización de tareas. }\end{array}$ \\
\hline & Imaginación & $\begin{array}{l}\text { - Presencia de planificación paso por paso para crear } \\
\text { una cadena de transformaciones proyectadas en } \\
\text { lenguaje externo. } \\
\text { - Características de la creatividad verbal orientada. } \\
\text { - Solución de problemas creativos reales presentes en } \\
\text { la escuela. }\end{array}$ \\
\hline
\end{tabular}

Fuente: elaboración propia.

\section{Análisis de resultados}

Los argumentos presentados por las maestras a través de las entrevistas a profundidad tienen considerables cambios de la fase uno a la fase tres. Con respecto a la primera categoría de análisis sobre "juego de roles", en la entrevista a profundidad de la fase uno, se obtuvieron las siguientes respuestas: 
Cuadro 4. Respuestas de las maestras a la categoría 1 "juego de roles en preescolar" en la fase uno.

Maestra $3^{\mathbf{o}} \mathbf{A}$
Reconoce que aplica actividades de juego, pero
no recuerda alguna teoría que lo sustente:
“..a veces aplico actividades de juego, sobre
todo actividades como cuentos motores o juegos
de psicomotricidad en el patio, en el salón yo sé
que juego, pero ahora que lo pienso, estoy
partiendo de mi experiencia, pero no tengo
claro qué autores hablan sobre el juego. Creo
que en la normal aprendí sobre las estrategias
básicas de aprendizaje, una de ellas es el
aprendizaje a través del juego, pero no
recuerdo más”.

Recuerda que ha recibido capacitación, pero no específica sobre el juego en preescolar:

"Ahora que estoy haciendo memoria, en los cursos de capacitación nos hablaron de la importancia del juego, de que los niños deben jugar en preescolar, pero no nos dijeron quién dice eso y por qué se dice, yo creo que es porque todos piensan que los niños solo juegan en preescolar y por eso ya dan por hecho que sabemos las razones".

Evoca que juega todo el tiempo, pero muestra confusión al explicar los momentos en los que aplica actividades de juego:

"Juego todo el tiempo, bueno, ahora ya no sé qué tipo de juego hago, solo sé que juego, por ejemplo, hacemos juegos en el patio para actividades motrices y deportivas, hacemos juegos de mesa como rompecabezas, loterías, pero cuando quiero que los niños aprendan algo concreto, no siempre juego, por ejemplo, si quiero que aprendan cosas sobre la naturaleza y el cuidado del medio ambiente no los pongo a jugar, los pongo a hacer experimentos, depende de lo que quiero lograr o del aprendizaje que estoy trabajando".
Reconoce que el juego es el medio por el que alcanza los aprendizajes, no hay evocación de teorías sobre el juego:

"El juego o actividad lúdica es el medio por el que trato de lograr los aprendizajes de los niños, por ejemplo, si estamos tratando aspectos de conteo, jugamos a la tiendita, así los niños se imaginan que venden y pagan, así a través del juego, los niños mejoran sus correspondencias al contar, no recuerdo ningún autor que hable sobre el juego, recuerdo a Piaget y a sus estadios, recuerdo que habla sobre las acciones matemáticas, supongo que eso tiene relación con el juego".

Reconoce que no se ha interesado por el tema de juego en preescolar:

"La verdad, hasta ahora que me lo preguntas lo analizo, nunca le había dado importancia real a entender el juego en preescolar, yo sé que ese es mi principal medio de enseñanza, pero no sé de donde tengo esa idea, a lo mejor en la normal lo escuché, bueno eso de que en preescolar se juega se escucha en todas partes, lo he leído en los libros de SEP".

Distingue dos tipos de juegos, los juegos libres y los juegos dirigidos:

"Algunas veces ocupo juego dirigido y otro juego libre. Cuando quiero observar el desarrollo social y emocional de los niños los dejo que jueguen libremente, yo solo los observo, por ejemplo, les doy material de construcción, pero siempre estoy al pendiente de que no se molesten o se lastimen. Cuando quiero que aprendan algo de matemáticas o estamos con algún aprendizaje de lenguaje y comunicación, aplico juego dirigido, ahí si yo organizo la actividad y oriento todo el tiempo a los niños". 
Manifiesta haber escuchado sobre el juego de roles, pero no tiene claridad sobre cómo se juega:

"Yo he escuchado mucho sobre el juego de roles, es cuando por ejemplo los niños juegan a la casita o a la tiendita, y se pone todo como si fuera de verdad, en el salón por ejemplo hemos jugado a la casita, unos niños son los papás, otros las mamás y otros los hijos, una vez que cada quien tiene un rol pues inicia el juego, cada niño va a hacer lo que ha visto que hacen en su propia casa, y así se juega, es como juego espontáneo".
Conoce el juego de roles, pero no distingue la teoría que lo sustenta:

"El juego de roles sirve para desarrollar habilidades socioemocionales, por ejemplo, la empatía, la regulación, la colaboración, etc. Son juegos donde a cada niño se le asigna un rol, como si fuera un actor de la sociedad y entonces se plantea una situación social, por ejemplo, si jugamos a ir al cine, pues se juega desde que se compran los boletos, se ve la película hasta que termina la función. No sé cuál es la teoría que sustenta el juego de roles, yo la verdad lo aplico poco porque no sé cómo organizarlo, yo veo que algunas otras maestras hacen juego de roles, pero veo que dejan que los niños hagan lo que quieren, así no me parece que sea correcto, por eso, mejor no lo hago, porque no se bien cómo se organiza”.

Como se puede observar, en el discurso de las maestras no hay presencia de un conocimiento sólido sobre la actividad de juego en la etapa preescolar, el juego es visto como una actividad de esparcimiento, incluso puede ser visto como una estrategia, idea que tiene su origen en los contenidos curriculares de las escuelas normales de educación preescolar en México, donde el juego es presentado como una de las seis estrategias básicas de aprendizaje, (SECRETARÍA DE EDUCACIÓN PÚBLICA, 2003, p. 88), sin embargo, al establecer puntos de reflexión con las maestras, se observa que han aplicado dichas actividades partiendo de una experiencia personal, lejos de postulados teóricos y metodológicos, es importante resaltar que ambas expresan en sus reflexiones que no han recibido una formación concreta en el plano profesional sobre el juego y sus implicaciones en la etapa preescolar.

Después de la capacitación sobre el enfoque histórico cultural y la aplicación de juego temático de roles bajo este postulado teórico (fase dos), las maestras mostraron discursos más sólidos, incluso, empleando referentes concretos y mostrando seguridad sobre las aportaciones de esta actividad en la etapa preescolar. Esto puede ser observado en el cuadro 5. 
Cuadro 5. Respuestas de las maestras a la categoría 1 "juego en preescolar" en la fase tres.

\section{Maestra $3^{\circ} \mathrm{A}$}

Reconoce al juego de roles como actividad rectora de la edad preescolar y ubica la perspectiva teórica que le da sustento:

"El juego es de gran importancia en la educación preescolar, no en el sentido simple de dejar jugar a los niños, sino en el reconocimiento de que el juego es la actividad rectora, desde el enfoque de Vigotsky, las actividades rectoras son las que propician el surgimiento de nuevas formaciones psicológicas en los niños, estas formaciones son las que de verdad garantizan el desarrollo, pero para que esto suceda, el juego debe ser organizado por la maestra, la maestra juega un rol bien importante porque, si no organiza el juego, si no da as instrucciones claras, si no acompaña las acciones de los niños, pues el juego de roles puede perder su riqueza".

\section{Maestra $3^{\circ} \mathrm{B}$}

Reconoce que el juego de roles es la actividad rectora de la edad preescolar, hay identificación del enfoque que sustenta el juego de roles:

"El juego de roles es la actividad rectora de la educación preescolar, desde el enfoque Histórico Cultural y ya después con las aportaciones de la Teoría de la Actividad esta idea quedó bien establecida. Me parece increíble que tengo muchos años de servicio y que después de tanto tiempo por fin haya entendido por qué el juego de roles es tan importante en preescolar. Si todas las maestras comprendiéramos que las nuevas formaciones psicológicas como la imaginación, la personalidad y la actividad voluntaria van a determinar el éxito de los niños en la primaria, pondríamos más atención en desarrollarlas, pero desafortunadamente esto no lo saben todas, y por eso nos equivocamos, además, en el programa de SEP tampoco se habla claramente de este tema, creo que las maestras deberíamos apostar más por la capacitación personal pero bien orientada, porque luego hacemos cursos solo por cumplir".
Con respecto a la recurrencia de las actividades de juego de roles, la maestra menciona que es necesario hacerlo de manera periódica:

"Después de la capacitación sobre el enfoque histórico cultural y después de que te vi Miss. (refiriéndose a la investigadora) aplicar juego de roles, quedé encantada, con ganas de jugar todo el tiempo, ahora que aplicamos juego de roles para la investigación tuvimos que sistematizar las sesiones pero, yo, con apoyo de la auxiliar hice juego de roles más veces, con los niños que tenían más dificultades, eso mismo hizo Miss E. (refiriéndose a la maestra de tercero B), dejaba a mis niños con la auxiliar y jugaba en el salón de cantos con diez niñitos que veo que no han avanzado en sus neoformaciones, sobre todo en la actividad voluntaria, y de verdad, hasta los papás ven los resultados. Deberíamos tener más tiempo para jugar roles en vez de invertir tanto en lo que dice el plan de estudios".
En relación a la recurrencia de las actividades de juego de roles, la maestra menciona que debería ser la actividad principal cada día:

"El juego de roles debería hacerse todos los días, pero hacerse de manera correcta, bien organizada, como se presenta en este enfoque, SEP nos pide que trabajemos los campos formativos, pero es más importante hacer el juego de roles, me puse de acuerdo con G. (refiriéndose a la maestra del tercero A) y aumentamos una vez más a la semana el juego de roles paro solo para los niños que consideramos requieren más apoyo, yo hice un equipo de ocho niños, porque estos niños son los que menos participan, hablan bien pero su expresión oral no es fluida, la verdad estoy sorprendida con estos niños, su lenguaje ahora es más amplio, son más seguros, lo cual denota los rasgos de la neoformación de la personalidad, además en general muestran mayor dominio de su propia conducta y eso es lo que se espera en la actividad voluntaria, podría hablar de cada niño pero no terminaría, lo que pi puedo decir es que en todos hay evidencias de mejora". 
Muestra conocimiento sobre el juego temático de roles:

"El juego de roles es muy amplio, lo importante es conocer las etapas del mismo, si uno no comprende que se debe respetar cada etapa del desarrollo del juego, se puede caer en el error de obviar por ejemplo el juego de representación material de roles y acciones, y como consecuencia, limitar el propio desempeño del niño ante una limitante de actividad que escapa de su comprensión. Lo interesante de este enfoque es que, además, se da lugar a la edad psicológica y no a la cronológica, en esta idea, pues es importante respetar el desarrollo de cada niño, nuestra tarea como maestras es observar ese desarrollo y darle las actividades justas para llegar a su zona de desarrollo próximo".
Muestra conocimiento sobre el juego temático de roles:

"Hay etapas que deben respetarse en el juego, no es entrar directo al juego de roles sociales, hay que pasar por las acciones en el plano material, los roles también inician siendo materiales, me parece bien interesante esto, leí el libro de juego de roles, algunas cosas todavía son muy elevadas para mí pero, ahora tengo clara la importancia del juego simbólico en el juego y su papel dentro del juego de roles, es que esto es muy amplio, las etapas del juego de roles, la importancia de la función simbólica, las neoformaciones que resultan de esta actividad, todo es muy claro, ojalá así nos hubieran explicado en la normal".

El discurso de las maestras es claro, se puede observar la asimilación de la postura teórica del enfoque histórico cultural, además de ello, es importante resaltar que sus argumentos demuestran reflexión personal y una motivación para generar cambios en su actividad educativa.

Con respecto a la categoría dos sobre las "neoformaciones en los niños de educación preescolar" las respuestas obtenidas a partir del discurso de las maestras sobre el estado de las neoformaciones de sus alumnos en la fase uno se muestra en el cuadro 6 . 
Cuadro 6. Respuestas de las maestras a la categoría 2 "neoformaciones en los niños de educación preescolar" en la fase uno.

\begin{tabular}{|c|c|c|c|}
\hline \multicolumn{2}{|c|}{ Categorías de análisis } & \multirow{2}{*}{$\begin{array}{l}\text { Maestra } 3^{\mathbf{o}} \mathbf{A} \\
\text { Poca iniciativa de los niños } \\
\text { en la propuesta de temas en } \\
\text { juegos. } \\
\text { Ejecución de actividades con } \\
\text { poca espontaneidad y } \\
\text { emotividad. }\end{array}$} & \multirow{2}{*}{$\begin{array}{l}\text { Maestra } 3^{\mathbf{o}} \mathbf{B} \\
\text { Iniciativa solo de algunos } \\
\text { niños en la propuesta de } \\
\text { temas en juegos. } \\
\text { Ejecución de actividades con } \\
\text { poca espontaneidad y } \\
\text { emotividad. }\end{array}$} \\
\hline $\begin{array}{l}\text { Personalida } \\
\text { d reflexiva }\end{array}$ & $\begin{array}{l}\text { Establecimiento } \\
\text { de motivos }\end{array}$ & & \\
\hline & $\begin{array}{l}\text { Comprensión de } \\
\text { normas de } \\
\text { comportamiento. }\end{array}$ & $\begin{array}{l}\text { Retroalimentación } \\
\text { constante y refuerzo de } \\
\text { normas de comportamiento. }\end{array}$ & $\begin{array}{l}\text { Retroalimentación constante } \\
\text { de normas de } \\
\text { comportamiento. }\end{array}$ \\
\hline & $\begin{array}{l}\text { Presencia del } \\
\text { sentido de } \\
\text { compasión por el } \\
\text { otro, deseo de } \\
\text { compartir y } \\
\text { ayudar. }\end{array}$ & $\begin{array}{l}\text { Poco desarrollo del sentido } \\
\text { de compasión, refuerzo } \\
\text { constante para desarrollar } \\
\text { acciones de ayuda y de } \\
\text { compartir. }\end{array}$ & $\begin{array}{l}\text { Nulo desarrollo del sentido } \\
\text { de compasión, refuerzo } \\
\text { constante para desarrollar } \\
\text { acciones de ayuda y de } \\
\text { compartir. }\end{array}$ \\
\hline & $\begin{array}{l}\text { Aspectos que } \\
\text { evidencian el } \\
\text { sentido personal } \\
\text { de los niños. }\end{array}$ & $\begin{array}{l}\text { Refuerzo verbal del adulto } \\
\text { para externar las } \\
\text { necesidades propias, gustos } \\
\text { y preferencias. }\end{array}$ & $\begin{array}{l}\text { Refuerzo verbal del adulto } \\
\text { para externar las } \\
\text { necesidades propias, gustos y } \\
\text { preferencias. }\end{array}$ \\
\hline $\begin{array}{l}\text { Actividad } \\
\text { voluntaria }\end{array}$ & $\begin{array}{l}\text { Subordinación } \\
\text { de las acciones } \\
\text { de los niños a la } \\
\text { instrucción del } \\
\text { adulto. }\end{array}$ & $\begin{array}{l}\text { Refuerzo constante en la } \\
\text { comprensión de } \\
\text { instrucciones. } \\
\text { Consolidación solo de } \\
\text { instrucciones breves. }\end{array}$ & $\begin{array}{l}\text { Refuerzo constante en la } \\
\text { comprensión de } \\
\text { instrucciones. } \\
\text { Consolidación de } \\
\text { instrucciones breves y dos } \\
\text { niños consolidan } \\
\text { instrucciones amplias. }\end{array}$ \\
\hline
\end{tabular}

Indicios sobre la autodirección de la propia conducta de los niños hacia objetivos establecidos y los

No hay presencia de la autodirección de la propia conducta de los niños hacia objetivos.
No hay presencia de la autodirección de la propia conducta de los niños hacia objetivos.

alcanzarlos. 
Uso del lenguaje en su función reguladora.
Constante regulación del comportamiento de los niños a través del lenguaje del adulto.

Dificultades en el manejo del lenguaje verbal y no verbal.

Diez niños focalizados con dificultades en la regulación de su comportamiento.
Constante regulación del comportamiento de los niños a través del lenguaje del adulto.

Ocho niños focalizados por presentar dificultades en la expresión oral.
No hay cumplimiento en la totalidad de las actividades que se solicitan a los niños. No hay verificación en la realización de tareas.
No hay cumplimiento en la totalidad de las actividades que se solicitan a los niños. No hay verificación en la realización de tareas.

\section{Imaginación Presencia de planificación para crear cadena de trasformaciones proyectada en lenguaje externo.}

Poca verbalización de los cambios realizados en las actividades.
Poca verbalización de los cambios realizados en las actividades.

\begin{tabular}{|c|c|c|}
\hline $\begin{array}{l}\text { Características } \\
\text { de la actividad } \\
\text { verbal } \\
\text { orientada. }\end{array}$ & $\begin{array}{l}\text { Imposibilidad en la } \\
\text { elaboración de argumentos } \\
\text { amplios en torno a } \\
\text { diferentes tópicos. } \\
\text { Poca iniciativa en la } \\
\text { invención de cuentos o en la } \\
\text { invención de historias. }\end{array}$ & $\begin{array}{l}\text { Imposibilidad en la } \\
\text { elaboración de argumentos } \\
\text { amplios en torno a diferentes } \\
\text { tópicos. } \\
\text { Poca iniciativa en la } \\
\text { invención de cuentos o en la } \\
\text { invención de historias. }\end{array}$ \\
\hline $\begin{array}{l}\text { Solución de } \\
\text { problemas } \\
\text { creativos } \\
\text { presentes en la } \\
\text { escuela }\end{array}$ & $\begin{array}{l}\text { Refuerzo verbal constante } \\
\text { del adulto para lograr } \\
\text { reflexiones en la solución de } \\
\text { problemas creativos } \\
\text { cotidianos. }\end{array}$ & $\begin{array}{l}\text { Refuerzo verbal constante del } \\
\text { adulto para lograr } \\
\text { reflexiones en la solución de } \\
\text { problemas creativos } \\
\text { cotidianos. }\end{array}$ \\
\hline
\end{tabular}

Las respuestas de las maestras a los indicadores de la categoría de análisis "neoformaciones en los niños de preescolar" de la fase uno, demuestran que los grupos de tercero, presentaban condiciones semejantes en cuando al estado de desarrollo de las neoformaciones de los niños a inicios de la investigación. En el discurso de las maestras se observa de manera constante el desconocimiento de que algunos aspectos de los indicadores son importantes en el desarrollo del niño. 
La maestra del tercer año grupo A, con respecto al indicador "establecimiento de motivos" de la neoformación personalidad, en la fase uno, comentó: "Nunca me había hecho consciente de la importancia de los motivos en los niños, siempre creí que estos se referían a la motivación que yo les doy para hacer las cosas, pero si aplico actividades de las que no estoy segura, ¿cómo me espero que los niños desarrollen motivos?". Este argumento por parte de la maestra nos da una clara muestra del desconocimiento que posee sobre la importancia de que los niños en edad preescolar, a través del establecimiento de motivos, logran subordinar conscientemente unas acciones a otras, aspecto que le permite adentrarse en las relaciones sociales, las normas y las reglas de comportamiento (ELKONIN, 1995), favoreciendo así no solo la neoformaciòn de la personalidad reflexiva sino de la actividad voluntaria.

Después de la aplicación de las sesiones de juego de roles, las respuestas cualitativas del discurso de las maestras tuvieron considerables cambios, en lo referente a las neoformaciones alcanzadas por sus alumnos, lo cual se refleja en el cuadro 7.

Cuadro 7. Respuestas de las maestras a la categoría 2 "neoformaciones en los niños de educación preescolar" en la fase tres.

\begin{tabular}{|c|c|c|c|}
\hline \multicolumn{2}{|c|}{ Categorías de análisis } & \multirow{2}{*}{$\begin{array}{c}\text { Maestra } 3^{\mathbf{o}} \mathbf{A} \\
\text { Iniciativa constante en la } \\
\text { propuesta de juegos de } \\
\text { roles, esta iniciativa } \\
\text { también se evidenció en } \\
\text { el resto de las actividades } \\
\text { escolares realizadas. }\end{array}$} & \multirow{2}{*}{$\begin{array}{c}\text { Maestra } 3^{\mathbf{o}} \mathbf{B} \\
\text { Iniciativa en la propuesta de } \\
\text { juegos de roles, el refuerzo } \\
\text { verbal del adulto sigue } \\
\text { teniendo presencia. }\end{array}$} \\
\hline \multirow{3}{*}{$\begin{array}{l}\text { Personalidad } \\
\text { reflexiva }\end{array}$} & \multirow{3}{*}{$\begin{array}{l}\text { Establecimiento } \\
\text { de motivos }\end{array}$} & & \\
\hline & & $\begin{array}{l}\text { Presencia de motivos en } \\
\text { realización de las } \\
\text { actividades de juego. }\end{array}$ & $\begin{array}{c}\text { Presencia de motivos en } \\
\text { realización de las actividades } \\
\text { de juego. }\end{array}$ \\
\hline & & $\begin{array}{l}\text { Ejecución de actividades } \\
\text { con presencia de } \\
\text { espontaneidad y } \\
\text { emotividad. }\end{array}$ & $\begin{array}{l}\text { Ejecución de actividades con } \\
\text { un alto grado de } \\
\text { espontaneidad y emotividad. }\end{array}$ \\
\hline
\end{tabular}


Inhibición de impulsos sometidos a motivos individuales.
Inhibición de impulsos sometidos a motivos individuales.
Subordinación de unas acciones a otras de acuerdo al motivo.

Poca participación del adulto en el refuerzo de las normas de

Comprensión de normas de comportamiento. comportamiento.
Subordinación de unas acciones a otras de acuerdo al motivo.

\begin{tabular}{ccc}
\hline & $\begin{array}{c}\text { Poca participación del } \\
\text { adulto en el refuerzo de } \\
\text { las normas de } \\
\begin{array}{c}\text { Comprensión de } \\
\text { normas de } \\
\text { comportamiento. }\end{array}\end{array}$ & $\begin{array}{c}\text { Poca participación del adulto } \\
\text { en el refuerzo de las normas } \\
\text { de comportamiento. }\end{array}$ \\
\cline { 2 - 3 } & $\begin{array}{c}\text { Progresión en la } \\
\text { asimilación de las normas } \\
\text { de comportamiento con } \\
\text { apoyo del adulto. }\end{array}$ & $\begin{array}{c}\text { Progresión en la asimilación } \\
\text { de las normas de } \\
\text { comportamiento. }\end{array}$ \\
\hline
\end{tabular}

Desarrollo del sentido de compasión, manifestación de acciones de ayuda mutua, colaboración y aceptación de acciones de compartir.
Desarrollo del sentido de la compasión y manifestación de acciones de ayuda entre niños del mismo grupo y con niños menores. compasión por el otro, deseo de compartir y ayudar.

Presencia de la colaboración y las acciones de compartir.

\begin{tabular}{|c|c|c|}
\hline \multirow[t]{2}{*}{$\begin{array}{l}\text { Aspectos que } \\
\text { evidencian el } \\
\text { sentido personal } \\
\text { de los niños. }\end{array}$} & $\begin{array}{l}\text { Reconocimiento y } \\
\text { verbalización de } \\
\text { necesidades propias, } \\
\text { explicación de } \\
\text { preferencias y } \\
\text { argumentación de gustos. }\end{array}$ & $\begin{array}{l}\text { Reconocimiento y } \\
\text { verbalización de necesidades } \\
\text { propias, explicación de } \\
\text { preferencias y argumentación } \\
\text { de gustos. }\end{array}$ \\
\hline & & $\begin{array}{l}\text { Exposición de motivos } \\
\text { personales ante los demás. }\end{array}$ \\
\hline $\begin{array}{l}\text { Subordinación } \\
\text { de las acciones } \\
\text { de los niños a la } \\
\text { instrucción del } \\
\text { adulto. }\end{array}$ & $\begin{array}{c}\text { Comprensión de } \\
\text { instrucciones amplias, en } \\
\text { algunos casos el } \\
\text { despliegue de la } \\
\text { explicación de la } \\
\text { instrucción es necesaria. }\end{array}$ & $\begin{array}{l}\text { Comprensión de instrucciones } \\
\text { amplias. }\end{array}$ \\
\hline
\end{tabular}

\section{Actividad voluntaria} os niños a la adulto. algunos casos el despliegue de la explicación de la amplias. 
Presencia del lenguaje externo de otros niños que contribuye a la explicación de la instrucción para ser comprendida por otros niños.
Presencia del lenguaje externo de otros niños que contribuye a la explicación de la instrucción para ser comprendida por otros niños.

Presencia de explicación de instrucciones amplias por parte de los niños.

Indicios sobre la autodirección de la propia conducta de los niños hacia objetivos establecidos y los medios para alcanzarlos.
Presencia de la autodirección de la conducta propia de cada niño hacia objetivos emanados de los motivos personales.

Presencia de acciones concretas para alcanzar objetivos (motivos).

Adecuada regulación del comportamiento de los niños a través del lenguaje del adulto.
Presencia de la autodirección de la conducta propia de cada niño hacia objetivos emanados de los motivos personales.

\section{Presencia de acciones concretas para alcanzar objetivos (motivos).}

Adecuada regulación del comportamiento de los niños a través del lenguaje del adulto.

\section{Uso del lenguaje en su función reguladora.}

\section{Presencia del lenguaje externo de los niños para regular su propio comportamiento.}

\section{Presencia del lenguaje externo de los niños para regular su propio comportamiento.}

Manejo adecuado de lenguaje verbal y no verbal.
Manejo adecuado de lenguaje verbal y no verbal.
Los diez niños focalizados mejoraron sobre la regulación de su propio comportamiento, tres de ellos requieren aun la regulación a través del lenguaje verbal del adulto, pero cada vez con menor participación de
Los ocho niños focalizados por dificultades de expresión oral, mostraron mayor dominio sobre la función comunicativa y generalizadora del lenguaje. éste. 


$\begin{array}{ccc} & \text { Con base en el } & \\ & \text { establecimiento de } & \text { Con base en el } \\ \text { motivos, hay mayor } & \text { presencia en el } & \text { establecimiento de motivos, } \\ \text { cumplimiento de las } & \text { cumplimiento de las tareas. } \\ \text { Control y } & \text { tareas. } & \\ \text { organización de } & & \end{array}$

tareas.

Se observa presencia de

la verificación en el juego

de roles y en la

realización de otras

actividades escolares.

Se observa presencia de la verificación en el juego de roles y en la realización de otras actividades escolares.

Presencia de la planificación paso a paso través del lenguaje

Presencia de planificación para crear cadena de trasformaciones proyectada en lenguaje externo. externo al explicar acciones a realizar en cada rol.

Presencia de la planificación paso a paso través del lenguaje externo al explicar acciones a realizar en cada rol.

En las actividades de dibujo dirigido se observaron transformaciones en las producciones de los niños, expresando a través de su lenguaje externo los elementos que planeaban incluir en su dibujo.

Presencia de argumentos en torno a situaciones sociales llevadas al juego de roles.

Presencia de argumentos en torno a situaciones sociales llevadas al juego de roles.

Características de la actividad verbal orientada.

Amplia iniciativa en la invención o recreación de situaciones sociales para realizar juego de roles e incluso para inventar cuentos.

Amplia iniciativa en la invención o recreación de situaciones sociales para realizar juego de roles e incluso para inventar cuentos.

Poca participación del lenguaje verbal del adulto para la solución de problemas cotidianos.

Poca participación del lenguaje verbal del adulto

Solución de problemas creativos presentes en la escuela para la solución de problemas cotidianos.
Iniciativa en la solución de problemas cotidianos.
Iniciativa en la solución de problemas cotidianos


Cualitativamente se pueden evidenciar los cambios en el desarrollo de las neoformaciones de personalidad reflexiva, actividad voluntaria e imaginación en los dos grupos de aplicación de juego de roles sociales. La orientación permanente que se dio a las maestras titulares de grupo, a los apoyos auxiliares y a las madres de familia que participaron en la investigación permitió hacer un ejercicio de constante revisión en la organización de los juegos, profundizando en aquellas acciones de orientación que centrarían sus beneficios en el desarrollo de las neoformaciónes de los niños de educación preescolar que participaron en la investigación. Sumado a lo anterior, se demostró la necesidad de poseer un dominio teórico para la organización de la actividad, en este caso, del juego de roles, como lo plantearon Vigotsky (1984, 1995, 1996), Elkonin (1989, 1995) y Leontiev (2010).

En el grupo de tercero A, los cambios más significativos se dieron en la neoformación de actividad voluntaria, la maestra de grupo refiere "Noto los mayores cambios en los niveles de comprensión de las instrucciones que doy, no solo en el juego de roles, sino en otras actividades escolares, los niños son capaces ahora de dirigir su propia conducta hacia objetivos establecidos, concretamente en el juego, los niños lograron asumir diferentes roles, respetando las acciones de cada uno de ellos, mi participación para recordarles lo que se podía o no hacer en determinada situación de juego cada vez fue menos necesaria. Los procesos de atención también mejoraron en el grupo, había juegos en los que no era necesaria mi orientación constante, los niños parecían jugar en una misma armonía". La reflexión de la maestra indica que hubo avances en el nivel de juego de roles, como señalan Solovieva y Quintanar (2012).

La maestra de tercer año grupo B consideró que los cambios más significativos se dieron en la neoformación de personalidad reflexiva "El juego de roles favoreció la creación de motivos en los niños, cada día ellos mismos mejoraron en la propuesta de nuevas situaciones de juego, mi 
refuerzo verbal para apoyarlos cada vez fue más esporádico, el motivo hacia el juego los hacia seguir el objetivo de la actividad, el grupo se volvió muy emotivo, los niños esperaban con ansias la llegada del día martes porque sabían que ese día hacíamos juego de roles, es más, desde el lunes ya empezaban a dar sus propuestas de juegos. Lo mejor fue ver como lograron controlar algunas acciones y ser fieles a su motivo de actividad, por ejemplo, había días en que ya era hora de salir al recreo, los niños disfrutan el recreo, pero preferían seguir en el juego de roles". Este argumento de la maestra también es indicativo de que los niños alcanzaron un nivel más avanzado de juego de roles (SOLOVIEVA; QUINTANAR, 2012), como se pudo vislumbrar también en el otro grupo.

\section{Conclusiones}

La investigación presentada demuestra la importancia del enfoque histórico cultural en la educación. Los sustentos de esta perspectiva teórica ofrecen a las maestras de educación preescolar la posibilidad de salir de la incertidumbre de las actividades consideradas como propias de la educación preescolar, sin sustento científico e iniciarse en la aplicación de juego temático de roles, considerado como actividad rectora de la edad preescolar (ELKONIN, 1989).

La principal aportación del juego temático de roles es que propicia la aparición de las neoformaciones básicas propias de la edad preescolar (VIGOTSKY 1984). La personalidad reflexiva, la actividad voluntaria y la imaginación, son tres neoformaciones importantes en el desarrollo psicológico del niño en edad preescolar, la aparición de estas nuevas formaciones psicológicas beneficiará el paso del niño al siguiente nivel educativo, la primaria, nivel en el que las actividades de lectura, escritura y cálculo no podrán darse sin las neoformaciones que debieron formarse en preescolar. 


\section{Referencias}

BONILLA, M; SOLOVIEVA, Y.; JIMÉNEZ, N. Valoración del nivel de desarrollo simbólico en la edad preescolar. Revista CES Psicología, v. 5, n. 2, p. 56 - 69, 2012. http://www.redalyc.org/articulo.oa?id=423539471005.

DAVIDOV, V. La teoría de la enseñanza que conduce al desarrollo. Moscú: INTER, 1996.

DAVIDOV, V. Tipos de generalización en la enseñanza. Moscú: Sociedad pedagógica de Rusia, 2000.

DYACHENKO, O. Acerca de las orientaciones básicas del desarrollo de la imaginación del preescolar. En Y., SOLOVIEVA Y.; L. QUINTANAR (Comp), Antología del desarrollo psicológico del niño en edad preescolar. México: Trillas, 2010. p. 197-204.

ELKONIN, D. Obras psicológicas escogidas. Moscú: Pedagogía, 1989.

ELKONIN, D. Desarrollo psicológico de las edades infantiles. Moscú: Academia de ciencias pedagógicas y sociales, 1995.

GARCÍA, M; SOLOVIEVA, Y.; QUINTANAR, L. El desarrollo de neoformaciones a través del juego y del cuento en niños preescolares, Cultura y educación, v. 25, n. 2, p.183 - 198, 2013. DOI:

https://www.tandfonline.com/doi/abs/10.1174/113564013806631255.

GÓMEZ, M. E. Panorama del sistema educativo mexicano desde la perspectiva de las políticas públicas. Innovación educativa, v. 17, n. 74, p. 143-163, 2017. http://www.scielo.org.mx/pdf/ie/v17n74/1665-2673-ie-17-74-00143.pdf.

GONZÁLEZ, C.; SOLOVIEVA, Y. Propuesta de método para el estudio de la formación de la función simbólica en la edad infantil. Tesis psicológica, v. 9, n. 2, p. $57-79,2014$. http://www.redalyc.org/articulo.oa?id=139039784005.

GONZÁLEZ, C.; SOLOVIEVA, Y. Impacto del juego de roles sociales en la formación de la función simbólica en preescolares, Psicología Universidad de Antioquia, v. 8, n. 2, p. 49 - 70, 2016. DOI:

http://dx.doi.org/10.17533/udea.rpsua.v8n2a04.

IZCARA, S. Manual de investigación cualitativa. México: Fontamara, 2014.

KEMMIS, S. La investigación acción. En A, Latorre, La investigación-acción, conocer y cambiar la práctica educativa 2005. p. 24, 35. 
LÁZARO, E; SOLOVIEVA, Y; CISNEROS, N.; QUINTANAR, L. Actividades de juego y cuento para el desarrollo psicológico del niño en preescolar. Revista Internacional Magisterio, v. 7, n. 37, p. 80-85, 2009.

LEONTIEV, A. El desarrollo psicológico del niño en edad preescolar. En Y., SOLOVIEVA Y.; L. QUINTANAR (Comp), Antología del desarrollo psicológico del niño en edad preescolar. México: Trillas, 2010. p. 17-26.

MENESES, M; MONGE, M. El juego en los niños: enfoque teórico. Revista educación, v. 25, n. 2, p. 113-124, 2001.

https://www.redalyc.org/articulo.oa?id=44025210.

PETROVSKY, A. El colectivo, la comunicación y el desarrollo de la personalidad. En I. Lliasov y V. Liaudis (Eds), Antología de la psicología pedagógica y de las edades. La Habana: Pueblo nuevo y educación, 1986. p. $161-166$.

SALMINA, N. y FILIMONOVA, O. Diagnóstico y corrección de la actividad voluntaria en niños preescolares y escolares. México: Universidad Autónoma de Tlaxcala, 2001.

SECRETARÍA DE EDUCACIÓN PÚBLICA. Programa de educación preescolar. México: SEP. 1979.

SECRETARÍA DE EDUCACIÓN PÚBLICA. Programa de educación preescolar. México: SEP. 1981.

SECRETARÍA DE EDUCACIÓN PÚBLICA. Programa de educación preescolar. México: SEP. 1992.

SECRETARÍA DE EDUCACIÓN PÚBLICA. Taller de diseño de actividades didácticas I y II. Programa y materiales de apoyo para el estudio. Licenciatura en Educación Preescolar. $5^{\circ}$ y $6^{\circ}$ semestre. México: SEP. 2003. p. 88.

SECRETARÍA DE EDUCACIÓN PÚBLICA. Programa de educación preescolar. México: SEP. 2004.

SECRETARÍA DE EDUCACIÓN PÚBLICA. Aprendizajes clave para la educación integral, educación preescolar. México: SEP. 2017.

SOLOVIEVA, Y; GONZÁLEZ, C; ROSAS, Y; MATA, E y MORALES, M. Resultados de investigación educativa desde el modelo histórico-cultural y la teoría de la actividad en la Universidad Iberoamericana de Puebla. Ensino Em Revista, v. 27, n. Especial, p. 1256-1272, 2020. DOI:

http://dx.doi.org/10.14393/ER-v27nEa2020-4. 
SOLOVIEVA, Y; QUINTANAR, L. El desarrollo del niño y los métodos de enseñanza. Elementos: ciencia y cultura, v. 17, n. 77, p. 9-13, 2010.

https://www.researchgate.net/publication/41619840_El_desarrollo_del_nino_y_los _metodos_de_ensenanza.

SOLOVIEVA, Y.; QUINTANAR, L. La actividad de juego en la edad preescolar. México: Trillas, 2012.

SOLOVIEVA, Y.; TEJEDA, L.; LAZARO, E.; QUINTANAR, L. Propuesta para el uso de juego de roles en la institución preescolar. Educación y filosofia.

Uberlandia, v. 29, n. 57, p. 153-174, 2015. DOI:

http://dx.doi.org/10.14393/REVEDFIL.issn.0102-6801.v29n57a2015-p153a174.

TALIZINA, N. La teoría de la actividad aplicada a la enseñanza. México:

Benemérita Universidad Autónoma de Puebla, 2009.

VIGOTSKY, L. Obras escogidas tomo IV. Moscú: Pedagogía, 1984.

VIGOTSKY, L. Obras escogidas, tomo III. Moscú: Visor, 1995.

VIGOTSKY, L. Obras psicológicas escogidas. Tomo IV. Moscú: Visor, 1996.

Recebido em agosto de 2020. Aprovado em dezembro de 2020. 